\title{
Determinación del Índice Facial Total y Cono Facial en Individuos Chilenos
}

\author{
Total Facial Index and Facial Cone in Chilean Individuals
}

Bustamante, F., ${ }^{* * * *}$; Alves, N.,**; Torres, C.****; Gatica, C. ${ }^{* * * * *}$ \& Olave, E.*****

BUSTAMANTE, F.; ALVES, N.; TORRES, C.; GATICA, C. \& OLAVE, E. Determinación del índice facial total y cono facial en individuos chilenos. Int. J. Morphol., 34(3):963-967, 2016.

RESUMEN: El estudio fue realizado en 68 estudiantes, adultos, Chilenos, de ambos sexos, con edades entre 17 y 32 años, oriundos de la Regiónde la Araucanía, Chile. En cada uno se registraron el diámetro vertical (Nasion-Gnation, Na-Gn) y diámetros transversales (bigoníaco y bicigomático, Go-Go y Cig-Cig, respectivamente) y se calculó el índice facial total (IFT). Para la obtención del cono facial se compararon los anchos Go-Go y Cig-Cig. Se analizó la relación entre: IFT v/s cono facial; IFT v/s sexo; cono facial v/s sexo. Para el análisis estadístico se utilizó las pruebas de chi-cuadrado, Pearson y coeficiente de correlación de Spearman, considerándose significativo $\mathrm{p}<0,05$. La altura de la cara $(\mathrm{Na}-\mathrm{Gn})$ fue de $117,5 \mathrm{~mm}$ en hombres y de 110,3 mm en mujeres; el ancho superior de la cara (Cig-Cig) fue de 126,9 mm en los hombres y de 124,4 $\mathrm{mm}$ en las mujeres. La altura facial es diferente para hombres y mujeres, siendo mayor en hombres. El IFT fue calculado a partir de las medidas registradas y se obtuvo lo siguiente: en hombres hubo 4 casos (5,9\%) euriprosopos, 4 (5,9\%) mesoprosopos y 17 (25\%) leptoprosopos; en mujeres hubo 12 casos (17,6 \%) euriprosopos, 12 $(17,6 \%)$ mesoprosopos y $19(27,9 \%)$ leptoprosopos. Concluimos que en individuos adultos chilenos la altura facial es diferente para hombres y mujeres, siendo mayor en hombres. El tipo leptoprosopo y la cara de forma triangular son más frecuentes en hombres. Los datos obtenidos son un aporte al conocimiento antropológico de un grupo de individuos del Sur de Chile.

PALABRAS CLAVE: Antropometría; Índice facial total; Individuos chilenos.

\section{INTRODUCCIÓN}

Los grupos étnicos tienen características similares en la gran mayoría de sus componentes, por lo que se ha podido caracterizar a ellos, en general. Tales características, sirven de referencia para la realización de estudios comparativos entre distintas razas aportando tanto al conocimiento antropológico como para la planificación del tratamiento clínico-quirúrgico y en la identificación forense.

La biometría de la cabeza en general y de la cara en particular, ha sido estudiada por diversos autores, quienes han aportado innumerables datos, tratando de caracterizar los diversos grupos étnicos.

El objetivo del presente estudio fue determinar en un grupo de individuos chilenos de la Región de la Araucanía, Chile, diámetros verticales y transversales de la cara para ob- tener el índice facial total (IFT) y el cono facial (CF). Mediante el IFT y utilizando la clasificación de Martin \& Saller (1957) se pudo clasificar los individuos en: hipereuriprosopos, europrosopos, mesoprosopos, leptoprosopos o hiperleptoprosopos. Además, se clasificó el aspecto de las caras de acuerdo al cono facial en: triangular o cuadrada.

\section{MATERIAL Y MÉTODO}

El estudio se realizó en 68 personas adultas, con edad entre 17 y 32 años, de ambos sexos (43 mujeres y 25 hombres), de la Región de la Araucanía, Chile. Los individuos aceptaron voluntariamente participar del estudio y firmaron el consentimiento informado. Este estudio ha sido aprobado

Facultad de Odontología, Universidad de La Frontera, Temuco, Chile.

* Centro de Investigación CIMA, Facultad de Odontología, Universidad de La Frontera, Temuco, Chile.

*** Centro de Investigación en Ciencias Odontologicas CICO, Facultad de Odontología, Universidad de La Frontera, Temuco, Chile.

**** Programa de Magíster en Ciencias, mención Morfología,Facultad de Medicina, Universidad de La Frontera, Temuco, Chile.

******* Facultad de Medicina, Universidad de La Frontera, Temuco, Chile.

Financiado por Proyecto DIUFRO No DI13-0061 
por el Comité Ético Científico de la Universidad de La Frontera con el No 013/13, acta de aprobación No 10/2013.

Para cada uno de ellos, se elaboró una ficha registrándose las siguientes mediciones faciales:

1. Diámetro vertical (altura facial): Nasion-Gnation

2. Diámetros transversales: Bigoníaco y bicigomático

Las mediciones fueron realizadas con un compás digital de corredera (cáliper) para el diámetro vertical y con un compás de espesor para los diámetros transversales.

El índice facial total fue determinado de acuerdo a:

$$
\text { IFT }=\underline{\text { Altura facial } \times 100}
$$

Tras obtener el valor del IFT se utilizó la clasificación de Martin \& Saller: hipereuriprosopo $(\leq 79,9)$, euriprosopo $(80,0$ - 84,9), mesoprosopo $(85,0$ - 89,9) leptoprosopo $(90,0$ - 94,9) e hiperleptoprosopo $(\geq 95,0)$.

El cono facial se obtuvo por comparación entre el ancho bicigomático y bigoníaco. Con valores muy cercanos, se consideró una cara cuadrilátera; con un ancho bigoníaco menor que el bicigomático se consideró la cara triangular.

Para el análisis estadístico se utilizaron las pruebas de chi-cuadrado y de Pearson para las variables cualitativas. Se utilizó el coeficiente de correlación de Spearman para cuantificar el grado de asociación entre las variables. Los datos fueron analizados con el software SPSS/PC 20.0. Se consideró estadísticamente significativo $\mathrm{p}<0,05$.

\section{RESULTADOS}

Los resultados de la mediciones de los diámetros faciales, vertical y transversales, obtenidos en 68 individuos chilenos se muestra en la Tabla I. Al comparar la altura facial, se encontro diferencias significativas según sexo $(\mathrm{p}=0,000)$ siendo mayor en hombres.

El IFT de acuerdo al sexo y el porcentaje relativo a la clasificación facial, son presentados en la Tabla II. El tipo leptoprosopo fue más frecuente que el euriprosopo y el mesoprosopo tanto para hombres $(\mathrm{p}=0,005 \mathrm{y} \mathrm{p}=0,005$, respectivamente) como para mujeres ( $\mathrm{p}=0,223 \mathrm{y} \mathrm{p}=0,157$, respectivamente), sin embargo, sólo en hombres hubo diferencia estadísticamente significativa. Ningún individuo fue clasificado como hipereuriprosopo o hiperleptoprosopo. Observamos que en individuos chilenos el IFT no es determinado por el sexo $(\mathrm{p}=0,165)$.

El aspecto del cono facial de acuerdo al sexo se muestra en la Tabla III. Observamos que la forma triangular fue la más frecuente, tanto para hombres $(\mathrm{p}=0,009)$ como para mujeres $(\mathrm{p}=0,101)$, sin embargo, sólo en hombres hubo diferencia estadísticamente significativa.

Tabla I. Promedio de los diámetros Nasion-Gnation, Bicigomático y Bigonial (mm).

\begin{tabular}{lllccc}
\hline Diámetros & Sexo & $\mathrm{n}$ & Promedio & Total & Desviacióón Estándar \\
\hline Na-Gn & Masculino & 25 & 117,5 & & 5,8 \\
& Femenino & 43 & 110,3 & 112,9 & 5,6 \\
Cig-Cig & Masculino & 25 & 126,9 & & 6,5 \\
& Femenino & 43 & 124,4 & 124,4 & 7,2 \\
Go-Go & Masculino & 25 & 101,9 & & 7,9 \\
& Femenino & 43 & 102,1 & 102,0 & 7,7 \\
\hline
\end{tabular}

Tabla II. Índice facial total de acuerdo al sexo.

\begin{tabular}{lcccccc}
\hline \multirow{2}{*}{ IFT } & \multicolumn{2}{c}{ Masculino } & \multicolumn{2}{c}{ Femenino } & \multicolumn{2}{c}{ Total } \\
\cline { 2 - 7 } & $\mathrm{N}^{\mathbf{0}}$ de casos & $\%$ del Total & $\mathrm{N}^{\circ}$ de casos & $\%$ del Total & $\mathrm{N}^{\circ}$ de casos & $\%$ del Total \\
\hline Hipereuriprosopo & 0 & 0 & 0 & 0 & 0 & 0 \\
Euriprosopo & 4 & 5,9 & 12 & 17,6 & 16 & 23,5 \\
Mesoprosopo & 4 & 5,9 & 12 & 17,6 & 16 & 23,5 \\
Leptoprosopo & 17 & 25 & 19 & 27,9 & 36 & 52,9 \\
Hiperleptoprosopo & 0 & 0 & 0 & 0 & 0 & 0 \\
Total & 25 & 36,8 & 43 & 63,2 & 68 & 100 \\
\hline
\end{tabular}


Tabla III. Aspecto del Cono Facial de acuerdo al sexo.

\begin{tabular}{lcccc}
\hline \multirow{2}{*}{ Forma } & \multicolumn{2}{c}{ Masculino } & \multicolumn{2}{c}{ Femenino } \\
\cline { 2 - 5 } Triangular & $\mathrm{N}^{\mathrm{o}}$ de casos & $\%$ & No de casos & Porcentaje \\
Cuadrilátera & 19 & 76 & 26 & 60,5 \\
\hline
\end{tabular}

No se encontró relación estadísticamente significativa entre las variables: IFT x sexo; cono facial x sexo; IFT x cono facial (Tabla IV).

\section{DISCUSIÓN}

Los rasgos faciales obtenidos a partir de morfometría facial pueden entregar información clave respecto a las características clínicamente relevantes que afectan el equilibrio facial como un todo y; por lo tanto; a la apariencia estética general del individuo.

En nuestro estudio aportamos datos sobre el índice facial total y el cono facial en individuos chilenos adultos. El índice facial total trata de la relación entre el diámetro vertical Nasio-Gnation y el diámetro bicigomático.

Los grupos étnicos tienen características similares en la gran mayoría de sus componentes, por lo que se ha podido caracterizar a ellos, en general, sin embargo, comparando con otros grupos, los diámetros registrados no son tan disímiles.

Los diámetros registrados en este estudio son semejantes a los reportados por Young et al. (1999), que en un grupo de individuos jóvenes obtuvieron una distancia Na-Gn de 118,0 mm ( \pm 9 ), valor con el que concordamos, principalmente con el grupo de individuos masculinos de nuestra serie cuyo valor promedio fue de $117,52 \mathrm{~mm}( \pm 5,8)$. En estudio realizado por del Sol (2006) en un grupo de individuos Mapuches de la Región Costera de la Araucanía, se obtuvo un promedio para el diámetro Na-Gn de $123,1 \mathrm{~mm}$, promedio levemente superior a los mencionados.

Por otra parte, Hossain et al., (2011) al estudiar esta variable en 832 estudiantes de sexo femenino en Japón, registraron un promedio de $114,4 \mathrm{~mm}( \pm 10,54)$ para el diámetro $\mathrm{Na}-\mathrm{Gn}$, resultado muy semejante al de Ngeow \& Aljunid (2009) quienes obtuvieron $115,6 \mathrm{~mm}( \pm 7,0)$ en jóvenes Malasios.

Con respecto al diámetro bicigomático, Young et al., informaron un registro levemente superior al de este estudio $(132 \mathrm{~mm}$ v/s $127 \mathrm{~mm}$ en hombres). Para este parámetro, del Sol obtuvo 143,5 $\mathrm{mm}( \pm 5,6)$, este último bastante superior a los 127 de la presente serie, diferencia dada tal vez por el grupo étnico a que pertenecen.
Tabla IV. Coeficiente de correlación Rho de Spearman (r) y valor p para las variables IFT, CF y sexo.

\begin{tabular}{lcc}
\hline Correlación entre variables & $r$ & valor $p$ \\
\hline IFT x sexo & 0,017 & 0,166 \\
CF x sexo & 0,141 & 0,240 \\
IFT x CF & $-0,06$ & 0,575 \\
\hline
\end{tabular}

Hossain et al., obtuvieron $138,49 \mathrm{~mm}$ para el diámetro bicigomático, este último resultado comparable a Young et al., y Wiganowska-Swiatkowska et al. (2013), que en un grupo de cantantes polacos obtuvieron un valor de $136,02 \mathrm{~mm}( \pm 13,43)$, así como Ngeow \& Aljunid que en un grupo de Malasios jóvenes determinaron 136,3 mm $( \pm 7,1)$.

Con los valores obtenidos y en base al índice prosópico de Martin \& Saller, nuestro estudio arrojó que un 52,9\% del total de individuos (hombres y mujeres) era leptoprosopo, teniendo una distribución semejante por sexo, diferente de lo informado por Mane et al., (2010) en un grupo de 100 individuos de la India (50 hombres y 50 mujeres), con edades semejantes a nuestra serie, obtuvieron que sólo un $23 \%$ tenían este índice y que el $48 \%$ de su muestra era hiperleptoprosopo. Otros dos grupos encontrados en nuestra muestra fueron europrosopo y mesoprosopo, en un mismo porcentaje $(23,5 \%)$, donde los autores mencionados determinaron para euriprosopo $10 \%$ y para mesoprosopo $15 \%$.

El estudio de del Sol en otro grupo de nuestra población, grupo étnico Mapuche, determinó que estos individuos tienen características mesoprosópicas con tendencia a euriprosópicas, con un $60 \%$ para los primeros y un $24 \%$ para los segundos, diferente a lo registrado en la presente investigación en relación al grupo principal, pero coincidiendo con el grupo euriprosopo.

La mesoprosopía encontrada por el autor mencionado, también ha sido señalada en otros grupos étnicos de Africa Central, esquimales, en hombres de la región de Haití y en individuos de la Región Sur de China (Lalouel, 1957; Gessain, 1958; Benoist, 1962; Olivier et al., 1966).

Los desequilibrios existentes en el diámetro vertical y anchura facial son característicos tanto en la mala oclusión clase II, como en clase III, alterando no solo la oclusión, sino también, la estética del paciente (Blanchette et al., 1966; Nanda, 1988; Franchi \& Baccetti, 2005). 
El cono facial corresponde a la proporción establecida entre los diámetros bicigomático y bigoniaco. Datos normativos para el cono facial aún no se han desarrollado, sin embargo, creemos que cuando estas medidas se presentan con valores muy cercanos, la cara presenta una forma cuadrada mientras que cuando el diámetro bigoniaco es menor en relación al bicigomático la cara es más triangular. De acuerdo con Sarver \& Jacobson (2007) esta forma facial triangular es la más atractiva.

Poco se sabe acerca de la etiología de la altura facial y los desequilibrios de ancho. La medición precisa de estos desequilibrios es esencial para el diagnóstico, la planificación del tratamiento y para el estudio de la etiología genética de fenotipos clínicos (Moreno Uribe et al., 2015). Anchura y altura facial se han estudiado en detalle enfermedades (Fonseca et al., 2014) como con la reper- cusión social (Wong et al., 2011). En ortodoncia, ancho y altura facial son también aspectos clave en el diagnóstico de mala oclusión y planificación del tratamiento (Moreno Uribe et al.). Además, se sabe que la planificación del tratamiento ortodóncico-quirúrgico comienza con el análisis morfológico y morfométrico de la cara, por lo tanto, es muy importante una comprensión conjunta de análisis facial y cefalométrico para determinar los objetivos terapéuticos.

Tomándose en cuenta los resultados obtenidos en nuestro estudio, podemos concluir que en individuos adultos chilenos la altura facial es diferente para hombres y mujeres, siendo mayor en hombres. Además, el tipo leptoprosopo y la cara de forma triangular son más frecuentes en los hombres.

BUSTAMANTE, F. ; ALVES, N. ;TORRES, C. ; GATICA, C. \& OLAVE, E. Total facial index and facial cone in chilean individuals. Int. J. Morphol., 34(3):963-967, 2016.

SUMMARY. The study was conducted on 68 students, Chilean adults, of both sexes, between 17 and 32 years old, originally from the Region de la Araucanía, Chile. The vertical diameter (Nasion-Gnation, Na-Gn) and transverse diameter (bigoníac and bizygomatic; Go-Go and Zig-Zig, respectively) were recorded and total facial index (TFI) was calculated. To obtain the facial cone widths Go-Go and Zig-Zig were compared. We analyzed the relationship between: TFI v/s facial cone; TFI v/s sex; facial cone v/s sex. For statistical analysis the chi-square test, Pearson and Spearman correlation coefficient was used, considering significant $\mathrm{p}$ $<0.05$. The face height ( $\mathrm{Na}-\mathrm{Gn})$ was $117.5 \mathrm{~mm}$ in men and $110.3 \mathrm{~mm}$ in women; the width of the upper face ( $\mathrm{Zg}-\mathrm{Zg}) \mathrm{was} 126.9 \mathrm{~mm}$ in men and $124.4 \mathrm{~mm}$ in women. The facial height is different for men and women, being higher in men. The TFI was calculated from the measurements recorded and the following is obtained: in men there were 4 cases (5.9\%) euriprosopic, 4 (5.9\%) mesoprosopic and $17(25 \%)$ leptoprosopic; in women there were 12 cases (17.6\%) euriprosopic, 12 (17.6\%) mesoprosopic and 19 (27.9\%) leptoprosopic. We hereby conclude that in Chilean adults the facial height is different for men and women, being higher in men. The leptoprosopic type and triangular face is more common for men. The data obtained is a contribution to anthropological knowledge of a group of individuals in the South of Chile.

KEY WORDS: Anthropometry; Total facial index; Chilean individuals.

\section{REFERENCIAS BIBLIOGRÁFICAS}

Benoist, J. Anthropologie physique de la population de l'ile de la tortue (Haïti). Contribution à l'étude de l'origine des noirs des Antilles Françaises. Bull. Mém. Soc. Anthrop. Paris, 3(3):31535, 1962.

Blanchette, M. E.; Nanda, R. S.; Currier, G. F.; Ghosh, J. \& Nanda, S. K. A longitudinal cephalometric study of the soft tissue profile of short- and long-face syndromes from 7 to 17 years. Am. J. Orthod. Dentofacial Orthop., 109(2):116-31, 1966.

del Sol, M. Facial index in mapuche individuals. Int. J. Morphol., 24(4):587-90, 2006.
Fonseca, F. R.; de Santana Sarmento, D. J.; Vasconcelos Medeiros P. F.; Diniz, D. N. \& dos Santos, M. T. Patients with mucopolysaccharidosis have tendencies towards vertical facial growth. J. Oral Maxillofac. Surg., 72(12):2539-46, 2014.

Franchi, L. \& Baccetti, T. Transverse maxillary deficiency in Class II and Class III malocclusions: a cephalometric and morphometric study on postero-anterior films. Orthod. Craniofac. Res., 8(1):21-8, 2005.

Gessain, R. Les Eskimo d'Angmassalik principaux caractères anthropologiques. L'Antropologie, 62(5-6):452-84, 1958. 
Hossain, M. G.; Saw, A.; Ohtsuki, F.; Lestrel, P. E. \& Kamarul, T. Change in facial shape in two cohorts of Japanese adult female students twenty years apart. Singapore Med. J., 52(11):81823, 2011.

Lalouel, J. Anthropométrie des Fang. Bull. Mém. Soc. Anthrop. Paris, 10(8):371-82, 1957.

Mane, D. R.; Kale, A. D.; Bhai, M. B. \& Hallikerimath, S. Anthropometric and anthroposcopic analysis of different shapes of faces in group of Indian population: a pilot study. $J$. Forensic Leg. Med., 17(8):421-5, 2010.

Martin, R. \& Saller, K. Lehrbuch der Anthropologie, in systematischer Darstellung. Bd 1. Stuttgart, Fisher, 1957.

Nanda, S. K. Patterns of vertical growth in the face. Am. J. Orthod. Dentofacial Orthop., 93(2):103-16, 1988.

Ngeow, W. C. \& Aljunid, S. T. Craniofacial anthropometric norms of Malays. Singapore Med. J., 50(5):525-8, 2009.

Olivier, G.; Phalippou, G. \& Tissier, H. Craniométrie des Chinois du Sud. Bull. Mém. Soc. Anthrop. Paris, 9(1):55-90, 1966.

Sarver, D. \& Jacobson, R. S. The aesthetic dentofacial analysis. Clin. Plast. Surg., 34(3):369-94, 2007.

Moreno Uribe, L. M.; Ray, A.; Blanchette, D. R; Dawson, D. V. \& Southard, T. E. Phenotype-genotype correlations of facial width and height proportions in patients with Class II malocclusion. Orthod. Craniofac. Res., 18 Suppl. 1:100-8, 2015.

Wyganowska-Swiatkowska, M.; Kowalkowska, I.; Mehr, K. \& Dabrowski, M. An anthropometric analysis of the head and face in vocal students. Folia Phoniatr. Logop., 65(3):136-42, 2013.

Wong, E. M.; Ormiston, M. E. \& Haselhuhn, M. P. A face only an investor could love: CEOs facial structure predicts their firms' financial performance. Psychol. Sci., 22(12):1478-83, 2011.

Young, D. V.; Rinchuse, D. J.; Pierce, C. J. \& Zullo, T. The craniofacial morphology of bruxers versus nonbruxers. Angle Orthod., 69(1):14-8,1999.

\author{
Dirección para Correspondencia: \\ Prof. Mg. Fabiola Bustamante \\ Departamento de Odontología Integral \\ Facultad de Odontología \\ Universidad de La Frontera \\ Temuco \\ CHILE
}

Email: fabiola.bustamante@ufrontera.cl

Recibido : 08-01-2016

Aceptado: 22-07-2016 